\title{
Existence of Mild Solutions of Abstract Fractional Differential Equations with Non-Instantaneous Impulsive Conditions
}

\author{
A. Anguraj and S. Kanjanadevi \\ PSG College of Arts and Science, Coimbatore-641 014, Tamil Nadu, India
}

\begin{abstract}
We consider a new class of fractional integro-differential equations with non instantaneous impulses. We establish an existence theorem for abstract fractional integro-differential equations with initial conditions under non instantaneous impulsive moments. The results are obtained by using the fixed point theorem for condensing map and resolvent operator.
\end{abstract}

\section{Introduction}

Fractional differential equations play the crucial and significant role in the field of science and engineering. Most importantly non-integer order differential equations have ability to describe the real behavior and memory effects of the system and processes. For more details about fractional differential equations and its applications refer the monographs and papers $[4,6,12,18,20]$.

The study of impulsive differential equations have more attention in recent years due to its applications. Most of the researchers dealt the differential equations with instantaneous impulses, which have been used to describe abrupt changes such as shocks, harvesting, and natural disasters, etc. For more details, we refer the reader to $[1,3,13,14,17]$.

Recently, in [8] E Hernández \& O’Regan introduced a non-instantaneous impulsive differential equations. In the model presented in [8], the impulses start abruptly at the point $t_{i}$ and their action continue on a finite time interval $\left[t_{i}, s_{i}\right]$. This type of problem motivates to study some certain dynamic change of evolution processes in pharmacotheraphy, [2, 7, 19,22].

On the other hand, we came to know from the semigroup theory that many authors used the concept of mild solutions inappropriately, see $[5,11,16]$. To make the concept of mild solutions more appropriate, E. Hernández et al [9] treated abstract differential equations with fractional derivatives in time, based on the well developed theory of resolvent operators for integral equations [21].

In this present work, we study the existence and uniqueness of fractional differential equations with non-instantaneous impulsive conditions of the form

$$
\begin{gathered}
{ }^{c} D^{\alpha} u(t)=A u(t)+f(t, \mathcal{B} u(t), u(t)), t \in\left(s_{i}, t_{i+1}\right], i=0,1, \cdots, N \\
u(t)=g_{i}(t, u(t)), t \in\left(t_{i}, s_{i}\right], i=1, \cdots, N \\
u(0)=u_{0}
\end{gathered}
$$


where $A$ is the infinitesimal generator of a $C_{0}$-semigroup of bounded linear operator $(S(t))_{t \geq 0}$ defined on a Banach space $(X,\|\|),. 0=t_{0}=s_{0}<t_{1}<s_{1}<\cdots<s_{N}<t_{N+1}=a$ are pre-fixed numbers, the functions $f:[0, a] \times X^{2} \rightarrow X, g_{i}:\left(t_{i}, s_{i}\right] \times X \rightarrow X, \quad$ for $\quad$ all $\quad i=1,2 \cdots, \quad N \quad$ are $\quad$ continuous, $\mathcal{B}: C([0, a] ; X) \rightarrow C([0, a] ; X)$ is given by $B x(t)=\int_{0}^{t} B(t, s) x(s) d s$ and $\{B(t, s): a \geq t \geq s \geq 0\}$ is a set of bounded linear operator on $X$ such that $B(t, \cdot) x \in C([0, t] ; X)$ and $B(\cdot, s) x \in C([s, a] ; X)$ for all $t, s \in[0, a]$ and each $x \in X .{ }^{c} D^{\alpha}$ is Caputo fractional derivative, where $0<\alpha<1$.

We use resolvent operator for integral equations to represent the mild solution of the system more appropriately and we use condensing map to prove the existence of the system.

\section{Preliminaries}

Let $\mathcal{L}(X, Y)$ be the space of bounded linear operators from the Banach space $X$ into the Banach space $Y$ endowed with the norm $\|\cdot\|_{\mathcal{L}(X, Y)}$ and we write simply $\mathcal{L}(X)$ when $X=Y$. Let $\|x\|_{\mathcal{D}}=\|x\|+\|A x\|$ where $\mathcal{D}$ is the domain of the operator $A$. In addition $B_{r}(x, X)$ represents the closed ball with center at $x$ and radius $r$ in $X . C([0, a] ; X)$ denotes the space of all the continuous functions from $[0, a]$ into $X$ with the sup-norm denoted by $\|\cdot\|_{C([0, a] ; X)}$.

We introduce the space $P C(X)$ which is formed by all the functions $u:[0, a] \rightarrow X$ such that $u($. is continuous at $t \neq t_{i}, u\left(t_{i}^{-}\right)=u\left(t_{i}\right)$ and $u\left(t_{i}^{+}\right)$exists for all $i=1, \cdots, N$, which is a Banach space with respect to the norm $\|u\|_{P C(X)}=\sup _{s \in[0, a]}\|u(s)\|$. For a function $u \in P C(X)$ and $i \in\{0,1, \cdots, N\}$, we introduce the function $u_{i} \in C\left(\left[t_{i}, t_{i+1}\right] ; X\right)$ given by

$$
\tilde{u}_{i}(t)=\left\{\begin{array}{l}
u(t), \text { for } t \in\left(t_{i}, t_{i+1}\right], \\
u\left(t_{i}^{+}\right), \text {for } t=t_{i}
\end{array}\right.
$$

In addition, for $E \subseteq P C(X)$ and $i \in\{0,1, \cdots, N\}$, we use the notation $E_{i}$ for the set $E_{i}=\left\{u_{i}: u \in E\right\}$. We note the following Ascoli-Arzela type criteria.

Lemma 2.1. [8] A set $E \subseteq P C(X)$ is relatively compact in $P C(X)$ if and only if each set $E_{i}$ is relatively compact in $C\left(\left[t_{i}, t_{i+1}\right] ; X\right)$.

We know that the definition of Caputo fractional derivative of order $\alpha>0$ of a function $u$ defined as follows:

$$
{ }^{c} D^{\alpha} u(t)=I^{n-\alpha} D^{n} u(t), n=\lceil\alpha\rceil
$$

where $I^{\alpha} u(t)=\frac{1}{\Gamma(\alpha)} \int_{a}^{t}(t-s)^{\alpha-1} u(s) d s$. Also, in general the Caputo derivative is a left inverse of $I^{\alpha}$ but not a right inverse, i.e., we have ${ }^{c} D^{\alpha} I^{\alpha} u=u$, and 


$$
I^{\alpha c} D^{\alpha} u(t)=u(t)-u(a), \text { for } 0<\alpha<1 .
$$

To establish our results, we assume that the following Volterra integral equation,

$$
u(t)=\frac{1}{\Gamma(\alpha)} \int_{0}^{t}(t-s)^{\alpha-1} A u(s)+f(t), t \in[0, a]
$$

has an associated resolvent operator $(S(t))_{t \geq 0}$ on $X$ and $f \in C([0, a] ; X)$.

Definition 2.1. [21] A family $(S(t))_{t \geq 0} \subset B(X)$ of bounded linear operators in $X$ is called resolvent for (2.2) (or solution operator for (2.2)), if the following conditions are satisfied

$S(t)$ is strongly continuous on $\mathbb{R}^{+}$and $S(0)=I$,

$S(t)$ commutes with $A$, which means that $S(t) \mathcal{D}(A) \subset \mathcal{D}(A)$ and $A S(t) x=S(t) A x$ for all $x \in \mathcal{D}(A)$ and $t \geq 0$;

The resolvent equation holds

$$
S(t) x=x+\int_{0}^{t} \frac{(t-s)^{\alpha-1}}{\Gamma(\alpha)} A S(s) x d s, \text { for all } x \in \mathcal{D}(A), t \geq 0 .
$$

In this paper, we always assume that $(S(t))_{t \geq 0}$ is analytic [21] and there is $\phi_{A} \in L_{l o c}^{1}\left(\mathbb{R}^{+}\right)$such that $\left\|S^{\prime}(t) x\right\| \leq \phi_{A}(t)\|x\|_{\mathcal{D}(A)}$ a.e. on $\mathbb{R}^{+}$for each $x \in \mathcal{D}(A)$.

Definition 2.2. [21] A function $u \in C([0, a] ; X)$ is called a mild solution of (2.2) on $[0, a]$ if $\frac{1}{\Gamma(\alpha)} \int_{0}^{t}(t-s)^{\alpha-1} u(s) d s \in C([0, a] ; \mathcal{D}(A))$ and

$$
u(t)=\frac{A}{\Gamma(\alpha)} \int_{0}^{t}(t-s)^{\alpha-1} u(s) d s+f(t) \text { on }[0, a] .
$$

Lemma 2.2. [21] Suppose (2.3) admits a resolvent $S(t)$ and let $f \in C([0, a] ; X)$. Then

if $u \in C([0, a] ; X)$ is a mild solution of $(2.2)$, then $\int_{0}^{t} S(t-s) f(s) d s$ is continuously differentiable on $[0, a]$ and

$$
u(t)=\frac{d}{d t} \int_{0}^{t} S(t-s) f(s) d s, t \in[0, a]
$$

in particular, mild solution of (2.2) are unique,

(ii) if $f \in C([0, a] ; \mathcal{D}(A))$ and $S(t)$ is differentiable then

$$
u(t)=f(t)+\int_{0}^{t} S^{\prime}(t-s) f(s) d s, t \in[0, a]
$$

is a mild solution of (2.2). 
In the following section, we prove the existence results by using condensing map fixed point theorem.

\section{Existence and Uniqueness of Solutions}

In this section, we prove the existence of mild solution of the system (1.1)-(1.3). First we rewrite fractional differential equations (1.1)-(1.3) is equivalent to the following fractional integral equation:

$$
u(t)=\left\{\begin{array}{l}
u_{0}+\frac{1}{\Gamma(\alpha)} \int_{0}^{t} \frac{A u(s)}{(t-s)^{1-\alpha}} d s+\frac{1}{\Gamma(\alpha)} \int_{0}^{t} \frac{f(s, \mathcal{B} u(s), u(s))}{(t-s)^{1-\alpha}} d s, t \in\left[0, t_{1}\right], \\
g_{i}\left(s_{i}, u\left(s_{i}\right)\right)+\frac{1}{\Gamma(\alpha)} \int_{s_{i}}^{t} \frac{A u(s)}{(t-s)^{1-\alpha}} d s+\frac{1}{\Gamma(\alpha)} \int_{s_{i}}^{t} \frac{f(s, \mathcal{B} u(s), u(s))}{(t-s)^{1-\alpha}} d s, t \in\left(s_{i}, t_{i+1}\right], \\
g_{i}(t, u(t)) t \in\left(t_{i}, s_{i}\right]
\end{array}\right.
$$

for each $i=1, \cdots, N$

Now, we introduce the concept of mild solution for (1.1)-(1.3) using the preliminary results on integral equation and Definition 2.2

Definition 3.3. A function $u \in P C([0, a] ; X)$ is said to be a mild solution of the integral equation (3) on $[0, a] \quad, \quad$ if $u(0)=u_{0}, u(t)=g_{i}(t, u(t)) \quad$ for $\quad$ all $\quad t \in\left(t_{i}, s_{i}\right], i=1, \cdots, N$, $\frac{1}{\Gamma(\alpha)} \int_{s_{i}}^{t} \frac{u(s)}{(t-s)^{1-\alpha}} d s \in \mathcal{D}(A), i=0,1, \cdots, N$ and

$$
u(t)=u_{0}+\frac{A}{\Gamma(\alpha)} \int_{0}^{t} \frac{u(s)}{(t-s)^{1-\alpha}} d s+\frac{1}{\Gamma(\alpha)} \int_{0}^{t} \frac{f(s, \mathcal{B} u(s), u(s))}{(t-s)^{1-\alpha}} d s,
$$

for all $t \in\left(0, t_{1}\right]$,

$$
u(t)=g_{i}\left(s_{i}, u\left(s_{i}\right)\right)+\frac{A}{\Gamma(\alpha)} \int_{s_{i}}^{t} \frac{u(s)}{(t-s)^{1-\alpha}} d s+\frac{1}{\Gamma(\alpha)} \int_{s_{i}}^{t} \frac{f(s, \mathcal{B} u(s), u(s))}{(t-s)^{1-\alpha}} d s
$$

for all $t \in\left(s_{i}, t_{i+1}\right], i=1, \cdots, N$.

Remark 3.1. In the remainder of this paper, $i_{c}$ denotes the inclusion map from $\mathcal{D}(A)$ into $X$ and we assume $K^{*}=\sup _{t \in[0, a]} \int_{0}^{t}\|B(t, s)\|_{L(X)} d s$ is finite.

Lemma 3.3. [9] Assume $S(t)$ is compact for all $t \geq 0$. Then $S^{\prime}(t)$ is compact for all $t \geq 0$ and the inclusion map $i_{c}: \mathcal{D}(A) \rightarrow X$ is compact.

For the existence of our main results we assume that the following hypothesis which are stated below:

(H1) The function $g_{i}:\left(t_{i}, s_{i}\right] \times X \rightarrow \mathcal{D}(A)$ is continuous and there exists 
$L_{g_{i}} \in C\left(\left(t_{i}, s_{i}\right] ; \mathbb{R}^{+}\right)$such that $\left\|g_{i}(t, u)-g_{i}(t, v)\right\|_{D(A)} \leq L_{g_{i}}(t)\|u-v\|$, for all $t \in\left(t_{i}, s_{i}\right], u, v \in X$.

(H2) There exists $m_{f} \in C\left([0, a] ; \mathbb{R}^{+}\right)$and a non-decreasing function $W_{f} \in C\left([0, \infty) ; \mathbb{R}^{+}\right)$such that $\|f(t, x, y)\|_{D} \leq m_{f}(t) W(\|x\|+\|y\|)$ for all $(t, x, y) \in[0, a] \times X \times X$.

In the following theorem, we establish the existence of a mild solution via a fixed point criterion for condensing operators. In the remainder of this paper, for $u \in P C(X)$ we use the notation $F_{u}$ for the function $F_{u}:[0, a] \rightarrow X$ given by

$$
F_{u}(t)=\frac{1}{\Gamma(\alpha)} \int_{s_{i}}^{t}(t-s)^{\alpha-1} f(s, \mathcal{B} u(s), u(s)) d s
$$

Theorem 3.1. Assume that $\left(H_{1}\right)-\left(H_{2}\right)$ are satisfied, $S(t)$ is compact for all $t>0, u_{0} \in \mathcal{D}(A)$, the functions $g_{i}(., 0)$ are bounded and

$$
\begin{aligned}
& \left(L_{g_{i}}+\left\|g_{i}\left(s_{i}, 0\right)\right\|+\frac{t_{i+1}^{\alpha}}{\alpha \Gamma(\alpha)}\left|m_{f}\right| \limsup _{r \rightarrow \infty} \frac{1}{r} W\left(\left(K^{*}+1\right) r\right)\right)\left(1+\left\|\varphi_{A}\right\|_{L^{1}\left(\left(s_{i}, t_{i+1}\right] ; \mathbb{R}^{+}\right)}\right)<1 \\
& \left(1+\frac{t_{1}^{\alpha}}{\alpha \Gamma(\alpha)}\left|m_{f}\right| \limsup _{r \rightarrow \infty} \frac{1}{r} W\left(\left(K^{*}+1\right) r\right)\right)\left(1+\left\|\varphi_{A}\right\|_{L^{1}\left(\left(0, t_{1}\right] ; \mathbb{R}^{+}\right)}\right)<1, \forall i=1, \cdots, N .
\end{aligned}
$$

Then there exists a mild solution $u \in P C(X)$ of the problem (1.1)-(1.3).

\section{Proof:}

Let the map $T: B_{r}(0, P C(X)) \rightarrow B_{r}(0, P C(X))$ be defined by using Lemma 2.2 as follows:

$$
T u(t)=\left\{\begin{array}{l}
u_{0}+\frac{1}{\Gamma(\alpha)} \int_{0}^{t} \frac{f(s, \mathcal{B} u(s), u(s))}{(t-s)^{1-\alpha}} d s \\
+\int_{0}^{t} S^{\prime}(t-s)\left[u_{0}+\frac{1}{\Gamma(\alpha)} \int_{0}^{s} \frac{f(\tau, \mathcal{B} u(\tau), u(\tau))}{(s-\tau)^{1-\alpha}} d \tau\right] d s, t \in\left[0, t_{1}\right] \\
g_{i}\left(s_{i}, u\left(s_{i}\right)\right)+\frac{1}{\Gamma(\alpha)} \int_{s_{i}}^{t} \frac{f(s, \mathcal{B} u(s), u(s))}{(t-s)^{1-\alpha}} d s \\
+\int_{s_{i}}^{t} S^{\prime}(t-s)\left[g_{i}\left(s_{i}, u\left(s_{i}\right)\right)+\frac{1}{\Gamma(\alpha)} \int_{s_{i}}^{s} \frac{f(\tau, \mathcal{B} u(\tau), u(\tau))}{(s-\tau)^{1-\alpha}} d \tau\right] d s, t \in\left(s_{i}, t_{i+1}\right] \\
g_{i}(t, u(t)), t \in\left(t_{i}, s_{i}\right]
\end{array}\right.
$$

for each $i=1, \cdots, N$.

Let $u \in P C([0, a] ; X)$ and for the assumption on $f, g_{i}$, we see that 


$$
\begin{aligned}
\int_{0}^{t} \| & S^{\prime}(t-s)\left(u_{0}+\frac{1}{\Gamma(\alpha)} \int_{0}^{s}(s-\tau)^{\alpha-1} f(\tau, \mathcal{B} u(\tau), u(\tau)) d \tau\right) \| d s \\
& \leq \int_{0}^{t} \varphi_{A}(t-s)\left\|u_{0}\right\|_{\mathcal{D}(A)} d s \\
& +\frac{1}{\Gamma(\alpha)} \int_{0}^{t} \varphi_{A}(t-s) \int_{0}^{s}(s-\tau)^{\alpha-1}\|f(\tau, \mathcal{B} u(\tau), u(\tau))\|_{\mathcal{D}(A)} d \tau d s \\
& \leq\left(\left\|u_{0}\right\|_{\mathcal{D}(A)}+\frac{t_{1}^{\alpha}}{\alpha \Gamma(\alpha)}\|f(\tau, \mathcal{B} u(\tau), u(\tau))\|_{\mathcal{D}(A)}\right)\left\|\varphi_{A}\right\|_{L^{1}\left(\left[0, t_{1}\right] ; \mathbb{R}^{+}\right)}
\end{aligned}
$$

which implies that $s \rightarrow \int_{0}^{t} S^{\prime}(t-s)\left(u_{0}+\frac{1}{\Gamma(\alpha)} \int_{0}^{s}(s-\tau)^{\alpha-1} f(\tau, \mathcal{B} u(\tau), u(\tau)) d \tau\right) d s$ is integrable on $\left[0, t_{1}\right]$.

Consider for the interval $\left(s_{i}, t_{i+1}\right], i=1, \cdots, N$,

$$
\begin{aligned}
& \int_{s_{i}}^{t}\left\|S^{\prime}(t-s)\left(g_{i}\left(s_{i}, t_{i+1}\right)+\frac{1}{\Gamma(\alpha)} \int_{s_{i}}^{s}(s-\tau)^{\alpha-1} f(\tau, \mathcal{B} u(\tau), u(\tau)) d \tau\right)\right\| d s \\
& \leq \int_{s_{i}}^{t} \varphi_{A}(t-s)\left\|g_{i}\left(s_{i}, t_{i+1}\right)\right\|_{\mathcal{D}(A)} d s \\
& +\frac{1}{\Gamma(\alpha)} \int_{s_{i}}^{t} \varphi_{A}(t-s) \int_{s_{i}}^{s}(s-\tau)^{\alpha-1}\|f(\tau, \mathcal{B} u(\tau), u(\tau))\|_{\mathcal{D}(A)} d \tau d s \\
& \leq\left(\left\|g_{i}\left(s_{i}, t_{i+1}\right)\right\|_{\mathcal{D}(A)}+\frac{t_{i+1}^{\alpha}}{\alpha \Gamma(\alpha)}\|f(\tau, \mathcal{B} u(\tau), u(\tau))\|_{\mathcal{D}(A)}\right)\left\|\varphi_{A}\right\|_{L^{1}\left(\left(s_{i}, t_{i+1}\right] ; \mathbb{R}^{+}\right)}
\end{aligned}
$$

then the function $s \rightarrow \int_{s_{i}}^{t} S^{\prime}(t-s)\left(g_{i}\left(s_{i}, u\left(s_{i}\right)\right)+\frac{1}{\Gamma(\alpha)} \int_{s_{i}}^{s}(s-\tau)^{\alpha-1} f(\tau, \mathcal{B} u(\tau), u(\tau)) d \tau\right) d s$ is integrable. This implies that $T$ is well defined.

Next, we prove that $T$ is a condensing map from $B_{r}(0, P C(X))$ into $B_{r}(0, P C(X))$.

To begin we show that $T$ has values in $B_{r}(0, P C(X))$. Let $u \in B_{r}(0, P C(X))$. For $i \geq 1$ and $t \in\left(s_{i}, t_{i+1}\right]$, we get

$$
\begin{aligned}
\|T u(t)\| & \leq L_{g_{i}}\left\|u\left(s_{i}\right)\right\|+\left\|g_{i}\left(s_{i}, 0\right)\right\|+\frac{1}{\Gamma(\alpha)} \int_{s_{i}}^{t} \frac{m_{f}(s)}{(t-s)^{1-\alpha}} W(\|\mathcal{B} u(s)\|+\|u(s)\|) d s \\
& +\int_{s_{i}}^{t} \varphi_{A}(t)\left(L_{g_{i}}\left\|u\left(s_{i}\right)\right\|+\left\|g_{i}\left(s_{i}, 0\right)\right\|\right. \\
& \left.+\frac{1}{\Gamma(\alpha)} \int_{s_{i}}^{s} \frac{m_{f}(\tau)}{(s-\tau)^{1-\alpha}} W(\|\mathcal{B} u(\tau)\|+\|u(\tau)\|) d \tau\right) d s \\
& \leq\left(L_{g_{i}} r+\left\|g_{i}\left(s_{i}, 0\right)\right\|+\frac{t_{i+1}^{\alpha}}{\alpha \Gamma(\alpha)}\left|m_{f}\right| W\left(\left(K^{*}+1\right) r\right)\right)\left(1+\left\|\varphi_{A}\right\|_{\left.L^{1}\left(s_{i}, t_{i+1}\right] ; \mathbb{R}^{+}\right)}\right)
\end{aligned}
$$

which implies that $\|T u\|_{C\left(\left(s_{i}, t_{i+1}\right] ; X\right)} \leq r$ for all $i \geq 1$. Arguing as above, we find that 


$$
\begin{aligned}
& \|T u\|_{C\left(\left(0, t_{1}\right] ; X\right)} \leq\left(\left\|u_{0}\right\|+\frac{t_{1}^{\alpha}}{\alpha \Gamma(\alpha)}\left|m_{f}\right| W\left(\left(K^{*}+1\right) r\right)\right)\left(1+\left\|\varphi_{A}\right\|_{L^{1}\left(\left(0, t_{1}\right] ; \mathbb{R}^{+}\right)}\right) \leq r \\
& \|T u\|_{\left.C\left(t_{i}, s_{i}\right] ; X\right)} \leq L_{g_{i}}\left\|u\left(s_{i}\right)\right\|+\left\|g_{i}\left(s_{i}, 0\right)\right\| \leq r
\end{aligned}
$$

from which we infer that $\|T u\|_{P C(X)} \leq r$ and $T$ has values in $B_{r}(0, P C(X))$.

To continue, we introduce the decomposition $T=\sum_{i=1}^{3} T_{i}$ where

$$
\begin{aligned}
& T_{1} u(t)=\left\{\begin{array}{l}
u_{0}+\int_{0}^{t} S^{\prime}(t-s) u_{0}, t \in\left[0, t_{1}\right] \\
g_{i}\left(s_{i}, u\left(s_{i}\right)\right)+\int_{s_{i}}^{t} S^{\prime}(t-s) g_{i}\left(s_{i}, u\left(s_{i}\right)\right), t \in\left(s_{i}, t_{i+1}\right] \\
g_{i}(t, u(t)), \quad t \in\left(t_{i}, s_{i}\right], i=1, \cdots, N
\end{array}\right. \\
& T_{2} u(t)=\left\{\begin{array}{l}
\frac{1}{\Gamma(\alpha)} \int_{s_{i}}^{t}(t-s)^{\alpha-1} f(s, \mathcal{B} u(s), u(s)) d s, t \in\left(s_{i}, t_{i+1}\right] \\
0, \quad \text { otherwise }
\end{array}\right. \\
& T_{3} u(t)=\left\{\begin{array}{l}
\int_{s_{i}}^{t} S^{\prime}(t-s) \frac{1}{\Gamma(\alpha)} \int_{s_{i}}^{s}(s-\tau)^{\alpha-1} f(\tau, \mathcal{B} u(\tau), u(\tau)) d \tau d s, t \in\left(s_{i}, t_{i+1}\right] \\
0, \quad \text { otherwise }
\end{array}\right.
\end{aligned}
$$

for each $i=0,1, \cdots, N$.

We divide the remainder of the proof into following steps

Step 1 The map $T_{1}$ is a contraction on $B_{r}(0, P C(X))$ From the properties of the function $g_{i}, i=1, \cdots, N$, it is easy to see that

$$
\begin{gathered}
\left\|T_{1} u-T_{1} v\right\|_{C\left(\left(s_{i}, t_{i+1}\right] ; X\right)} \leq L_{g_{i}}\left(1+\left\|\varphi_{A}\right\|_{\left.L^{1}\left(s_{i}, t_{i+1}\right] ; \mathbb{R}^{+}\right)}\right)\|u-v\|_{P C(X)} \\
\left\|T_{1} u-T_{1} v\right\|_{C\left(\left(t_{i}, s_{i}\right] ; X\right)} \leq L_{g_{i}}\|u-v\|_{P C(X)}, u, v \in P C(X) .
\end{gathered}
$$

which implies that $\left\|T_{1} u-T_{1} v\right\|_{P C(X)} \leq \Theta\|u-v\|_{P C(X)}$ and $T_{1}$ is a contraction on $B_{r}(0, P C(X))$, because $\Theta=\max _{i=1, \cdots, N}\left\{L_{g_{i}}\left(1+\left\|\varphi_{A}\right\|_{L^{1}\left(\left(s_{i}, t_{i+1}\right] \mathbb{R}^{+}\right)}\right)\right\}<1$.

Step 2 The map $T_{2}$ is completely continuous on $B_{r}(0, P C(X))$ It is easy to see that $T_{2}$ is continuous. Next we prove that $T_{2}$ is a compact operator on $B_{r}(0, P C(X))$.

Let $s_{i}<\varepsilon<t_{i+1}, i=0,1, \cdots, N$. From the mean value theorem for the Bochner integral ([15], Lemma 2.1.3), for $u \in B_{r}(0, P C(X))$ and $\varepsilon \leq t \leq t_{i+1}$, we see that 


$$
\begin{aligned}
T_{2} u(t) & =\frac{1}{\Gamma(\alpha)} \int_{t-\varepsilon}^{t} \frac{f(s, \mathcal{B} u(s), u(s))}{(t-s)^{1-\alpha}} d s+\frac{1}{\Gamma(\alpha)} \int_{s_{i}}^{t-\varepsilon} \frac{f(s, \mathcal{B} u(s), u(s))}{(t-s)^{1-\alpha}} d s \\
& \in B_{\frac{C_{1}^{\alpha} \alpha^{\alpha}}{\alpha \Gamma(\alpha)}}(0, X)+\frac{\left(t-\varepsilon-s_{i}\right)}{\Gamma(\alpha)} \overline{c o\left(\left\{(t-s)^{1-\alpha} f(s, \mathcal{B} u(s), u(s)): s \in\left[s_{i}, t-\varepsilon\right]\right\}\right)}
\end{aligned}
$$

from the above we infer that

$$
\left\{T_{2} u(s): u \in B_{r}(0, P C(X)), s \in\left[\varepsilon, t_{i+1}\right]\right\} \subset B_{\frac{C_{1} \varepsilon^{\alpha}}{\alpha \Gamma(\alpha)}}(0, X)+K_{\varepsilon},
$$

where $K_{\varepsilon}$ is a compact subset of $X$. Moreover, by using that

$$
\left\{T_{2} u(s): u \in B_{r}(0, P C(X)), s \in\left[s_{i}, \varepsilon\right]\right\} \subset B_{\frac{C_{1} \varepsilon^{\alpha}}{\alpha \Gamma(\alpha)}}(0, X)
$$

we find that

$$
\left\{T_{2} u(s): u \in B_{r}(0, P C(X)), s \in\left[s_{i}, t_{i+1}\right]\right\} \subset B_{\frac{c_{1} \varepsilon^{\alpha}}{\alpha \Gamma(\alpha)}}(0, X) \cup\left(B_{\frac{c_{1} \varepsilon^{\alpha}}{\alpha \Gamma(\alpha)}}(0, X)+K_{\varepsilon}\right),
$$

which permit us to conclude that $\left\{T_{2} u(s): u \in B_{r}(0, P C(X)), s \in\left[s_{i}, t_{i+1}\right]\right\}$ is relatively compact in $\mathrm{X}$. Since $\frac{C_{1} \varepsilon^{\alpha}}{\alpha \Gamma(\alpha)} \rightarrow 0$ as $\varepsilon \rightarrow 0$.

On the other hand, we have that

$$
\left\|T_{2} u(t+h)-T_{2} u(t)\right\| \leq \frac{2 C_{1}}{\alpha \Gamma(\alpha)} h^{\alpha}
$$

for all $u \in B_{r}(0, P C(X))$, which implies that $T_{2} B_{r}(0, P C(X))$ is equicontinuous. This completes the proof that $T_{2}$ completely continuous.

Step 3. The map $T_{3}$ is completely continuous.

First, we prove that $\left\{T_{3} u(t): u \in B_{r}(0, P C(X))\right\}$ is relatively compact in $X$ for all $t \in\left(s_{i}, t_{i+1}\right], i=0,1, \cdots, N$.

Let $s_{i}<t \leq t_{i+1}$ and $C_{\varepsilon}=\left|m_{f}\right| W\left(\left(K^{*}+1\right) r\right)\left\|\varphi_{A}\right\|_{L^{1}\left(\left[s_{i}, \varepsilon\right]\right)}$. We know that from the above step, the set $U=\left\{F_{u}(s): s \in\left(s_{i}, t_{i+1}\right], u \in B_{r}(0, P C(X))\right\}$ is relatively compact in $X$.

If $u \in B_{r}(0, P C(X))$, from the mean value theorem for the Bochner integral we get

$$
\begin{aligned}
T_{3} u(t) & =\int_{s_{i}}^{t-\varepsilon} S^{\prime}(t-s) F_{u}(s) d s+\int_{t-\varepsilon}^{t} S^{\prime}(t-s) F_{u}(s) d s \\
& \subset\left(t-\varepsilon-s_{i}\right) \overline{c o\left(\left\{S^{\prime}(s) u: s \in[\varepsilon, t], u \in \bar{U}\right\}\right)}+B_{C_{\varepsilon}}(0, X)
\end{aligned}
$$

and hence, $\left\{T_{3} u(t): u \in B_{r}(0, P C(X))\right\} \subset K_{\varepsilon}+B_{C_{\varepsilon}}(0, X)$ where $K_{\varepsilon}$ is compact and $C_{\varepsilon} \rightarrow 0$ as 
$\varepsilon \rightarrow 0$.

This proves that $\left\{T_{3} u(t): u \in B_{r}(0, P C(X))\right\}$ is relatively compact in $X$.

Now, we prove that the set of functions $T_{3} B_{r}(\overline{0, P C}(X))$ is an equicontinuous subset of $C\left(\left(s_{i}, t_{i+1}\right] ; X\right)$.

Assume $t \in\left(s_{i}, t_{i+1}\right)$. Since $S^{\prime}(.) \in C((0, a] ; X)$, for a given $\varepsilon>0,\left\|S^{\prime}(t)-S^{\prime}(s)\right\|_{L(X)} \leq \varepsilon$ whenever $\|t-s\|_{\delta}$ where $s_{i}<s<t<t_{i+1}, i \geq 1$ and.

For $u \in T_{3} B_{r}(0, P C(X))$ and $0<h<\varepsilon$ such that $t+h \leq t_{i+1}$ we get

$$
\begin{aligned}
\| T_{3} \overline{u(t+h)}-T_{3} \widetilde{u(t)} & \|=\| T_{3} u(t+h)-T_{3} u(t) \| \\
& =\left\|\int_{s_{i}}^{t+h} S^{\prime}(t+h-s) F_{u}(s) d s-\int_{s_{i}}^{t} S^{\prime}(t-s) F_{u}(s) d s\right\| \\
& \leq \int_{0}^{t-s_{i}}\left\|S^{\prime}(\tau+h)-S^{\prime}(\tau)\right\|\left\|F_{u}(t+\tau)\right\| d \tau+\int_{0}^{h} \varphi_{A}(t)\left\|F_{u}(t+\tau)\right\| d \tau \\
& \leq \frac{C_{1} t_{i+1}^{\alpha}}{\alpha \Gamma(\alpha)}\left(\varepsilon+\left\|\varphi_{A}\right\|_{L^{1}\left((0, h) ; \mathbb{R}^{+}\right)}\right)
\end{aligned}
$$

which proves that $T_{3} B_{r}(\widetilde{0, P C}(X))$ is right equicontinuous at $t$.

Proceeding as above, for $t=s_{i}$ and $h>0$ with $s_{i}+h<t_{i+1}$ we have that

$$
\begin{aligned}
\left\|T_{3} \overline{u\left(s_{i}+h\right)}-T_{3} \widetilde{u\left(s_{i}\right)}\right\| & =\left\|\int_{s_{i}}^{s_{i}+h} S^{\prime}(t+h-s) F_{u}(s) d s\right\| \\
& \leq \frac{C_{1} t_{i}^{\alpha}}{\alpha \Gamma(\alpha)}\left\|\varphi_{A}\right\|_{L^{1}\left(\left[s_{i}, s_{i}+h\right]\right)}
\end{aligned}
$$

which implies that $T_{3} B_{r}(\widetilde{0, P C}(X))$ is right equicontinuous at $s_{i}$. A similar procedure permits us to show that $T_{3} B_{r}(\widetilde{0, P C}(X))$ is left equicontinuous at $t \in\left(s_{i}, t_{i+1}\right]$. This completes the proof that the set $T_{3} B_{r}(\widetilde{0, P C}(X))$ is equicontinuous.

From the above steps and Lemma 2.1 it follows that $T_{1}$ is a contraction, $T_{2}, T_{3}$ are completely continuous and $T=T_{1}+T_{2}+T_{3}$ is a condensing operator from $B_{r}(0, P C(X))$ into $B_{r}(0, P C(X))$. Finally, from ([15], Theorem 4.3.2) we infer there exists a mild solution of (1.1)-(1.3).

\section{Application}

To study the existence of solutions for the partial differential system with fractional temporal derivative, we may take the space $X=L^{2}([0, \pi])$ and $A$ be the operator given by $A x=x^{\prime \prime}$ with domain 
$\mathcal{D}(A)=\left\{x \in X: x^{\prime \prime} \in X, x(0)=x(\pi)=0\right\}$. It is well-known that $A$ is the infinitesimal generator of an analytic semigroup $(T(t))_{t \geq 0}$ on $X$.

Furthermore, $A$ has discrete spectrum with eigenvalues of the form $-n^{2}, n \in \mathbb{N}$, and corresponding normalized eigenfunctions given by $z_{n}(\zeta)=\left(\frac{2}{\pi}\right)^{1 / 2} \sin (n \zeta)$. In addition, $\left\{z_{n}: n \in \mathbb{N}\right\}$ is an orthonormal basis for $X, T(t) x=\sum_{n=1}^{\infty} e^{-n^{2} t}<x, z_{n}>z_{n}$ for all $x \in X$ and for every $t>0$. From these expressions it follows that $(T(t))_{t \geq 0}$ is a uniformly bounded compact semigroup, so that, $R(\lambda, A)=(\lambda-A)^{-1}$ is a compact operator for all $\lambda \in \rho(A)$.

Now, consider the following partial fractional impulsive and nonlocal systems:

$$
\begin{gathered}
{ }^{c} D^{\alpha} u(t, \zeta)=\frac{\partial^{2}}{\partial \zeta^{2}} u(t, \zeta)+\int_{0}^{t} a_{1}(t-s) u(s, \zeta) d s+a_{2}(t, u(t, \zeta)), \\
(t, \zeta) \in \cup_{i=1}^{N}\left(s_{i}, t_{i+1}\right] \times[0, \pi], \\
u(t, 0)=u(t, \pi)=0, t \in[0, a], \\
u(0, \zeta)=z(\zeta), \zeta \in[0, \pi], \\
u(t, \zeta)=G_{i}(t, u(t, \zeta)), \zeta \in[0, \pi], t \in\left(t_{i}, s_{i}\right], i=1, \cdots, N .
\end{gathered}
$$

where $\quad 0=t_{0}=s_{0}<t_{1}<s_{1}<\cdots<t_{N}<s_{N}<t_{N+1}=a \quad$ are fixed real numbers, $\alpha \in(0,1), \quad z \in X, a_{i} \in C([0, a] \times R ; R), G_{i} \in C\left(\left(t_{i}, s_{i}\right] \times \mathbb{R} ; \mathbb{R}\right)$ for all $i=1, \cdots, N$.

To represent the fractional impulse system (4.1)-(4.4) in the abstract form (1.1)-(1.3), we consider the functions $f:[0, a] \times X^{2} \rightarrow X, g_{i}:\left(t_{i}, s_{i}\right] \times X \rightarrow X, \quad B(t, s): X \rightarrow X, B: C([0, a] ; X) \rightarrow X$ defined by

$$
f(t, u, v)(\zeta)=\int_{0}^{t} a_{1}(t-s) u(s, \zeta) d s+a_{2}(t, u(t, \zeta)), g_{i}(t, u)(\zeta)=G_{i}(t, u(t, \zeta))
$$

It is easy to see that $G_{i}\left(s_{i}, 0\right), B(t, s)$ and $B($.$) are bounded linear operators, \|\mathcal{B}\| \leq\left\|a_{1}\right\|_{L^{1}[0, a]}$

Next, from [21] we know that the integral equation,

$$
u(t)=\frac{1}{\Gamma(\alpha)} \int_{0}^{t}(t-s)^{\alpha-1)} A u(s) d s, s \geq 0
$$

has an associated analytic resolvent operator $(S(t)) t \geq 0$ on $X$ which is given by 
Existence of Mild Solutions of Abstract Fractional Differential Equations with Non-Instantaneous Impulsive Conditions

$$
S(t)=\left\{\begin{array}{l}
\frac{1}{2 \pi i} \int_{\Gamma_{r, \theta}} e^{\lambda t} \lambda^{\alpha-1}\left(\lambda^{\alpha}-A\right)^{-1} d \lambda, t>0, \\
I, \quad t=0,
\end{array}\right.
$$

where $\Gamma_{r, \theta}$ denotes the contour consisting of the rays $\left\{r e^{i \theta}: r \geq 0\right\}$ and $\left\{r e^{-i \theta}: r \geq 0\right\}$ for some some $\theta \in(\pi, \pi / 2)$.

Now, $u \in P C(X)$ is a mild solution of (4.1)-(4.4), if $u($.$) is a mild solution of the associated abstract$ problem (1.1)-(1.3).

Proposition 4.1. If $G_{i}$ are Lipschitz functions with Lipschitz constant $L_{G_{i}}$, and

$$
\begin{aligned}
& \left(L_{G_{i}}+\left\|G_{i}\left(s_{i}, 0\right)\right\|+\frac{t_{i+1}^{\alpha}}{\alpha \Gamma(\alpha)}\left|m_{f}\right| \limsup _{r \rightarrow \infty} \frac{1}{r} W\left(\left(K^{*}+1\right) r\right)\right)\left(1+\left\|\phi_{A}\right\|_{L^{1}\left(\left(s_{i}, t_{i+1}\right] ; \mathbb{R}^{+}\right)}\right)<1 \\
& \left(1+\frac{t_{1}^{\alpha}}{\alpha \Gamma(\alpha)}\left|m_{f}\right| \limsup _{r \rightarrow \infty} \frac{1}{r} W\left(\left(K^{*}+1\right) r\right)\right)\left(1+\left\|\phi_{A}\right\|_{L^{1}\left(\left(0, t_{1}\right] ; \mathbb{R}^{+}\right)}\right)<1, \forall i=1, \cdots, N .
\end{aligned}
$$

then there exists a mild solution $u \in P C(X)$ of (4.1)-(4.4).

\section{References}

A. Anguraj, K. Karthikeyan, Existence of solutions for impulsive neutral functional differential equations with nonlocal conditions, Nonlinear Anal., 70 (2009), 2717-2721.

A. Anguraj, M. Lathamaheshwari, Existence results for fractional differential equations with infinite delay and interval impulsive coonditions, Malaya J. Mat. 2 (1) (2014) 16-23.

A. Anguraj, M. Mallika Arjunan, Existence and uniqueness of mild and classical solutions of impulsive evolution equations, Elect. J. Diff. Eqns., 111 (2005), 1-8.

A. Anguraj, P. Karthikeyan, G.M. N'uerekata, Nonlocal cauchy problem for some fractional abstract integrodifferential equations in Banach spaces, Commun. in Math. Anal., 1 (2009), 31-35.

M. Belmekki, M. Benchohra, Existence results for fractional order semilinear functional differential equations with nondense domain, Nonlinear Anal., 72 (2010), 925-932.

D. Delbosco, L. Rodino, Existence and uniqueness for a nonlinear fractional differential equations, J. of Math. Anal. Appl., 204 (1996), 609-625.

Ganga Ram Gautam, Jaydev Dabas, Existence result of fractional functional integro-differential equation with not instantaneous impulse, Int. J. Adv. Appl. Math. and Mech. 1 (3) (2014) 11-21

Eduardo Hernández, Donal O'Regan, On a new class of abstract impulsive differential equations, Proceedings of the American Mathematical Society, 141 (2013), 1641-1649.

Eduardo Hernández, Donal O’Regan, Krishnan Balachandran, On recent developments in the theory of abstract differential equations with fractional derivatives, Nonlinear Analysis, 73 (2010), 3462-3471.

Eduardo Hernández, Donal O’Regan, Krishnan Balachandran, Existence results for abstract fractional differential equations with nonlocal conditions via resolvent operators, Indagationes mathematicae, 24 (2013), 68-82.

O.K. Jaradat, A. Al-Omari, S. Momani, Existence of mild solutions for fractional semilinear initial value problems, Nonlinear Anal., 69 (9) (2008),3153-3159.

A.A. Kilbas, H.M. Srivastava, J.J. Trujillo, Theory and application of fractional differential equations, North-Holland, Amsterdam, 2006.

V. Lakshmikantham, DD. Bainov, P.S. Simeonov, Theory of impulsive differential equations, World Scientific, Singapore, 1989.

James H. Liu, Nonlinear impulsive evolution equations, Dynam. Contin. Discrete Impuls. Systems 6 (1999), 77-85.

R.H. Martin, Nonlinear operators and differential equations in Banach spaces, Robert E. Krieger publ. co., Florida, 1987. 
G.M. Mophou, Existence and uniqueness of mild solutions to impulsive fractional differential equations, Nonlinear Anal., 72 (2010), 1604-1615.

J.J. Nieto , D. O’Regan, Variational approach to impulsive differential equations, Nonlinear Anal., Real World Appl. 10 (2009), 680-690.

D.N. Pandey, A. Ujlayan, D. Bahuguna, On a solution to fractional order integrodifferential equations with analytic semigroups, Nonlinear Anal., 71 (2009), 3690-3698.

M. Pierri, Donal O'Regan, V. Rolink, Existence of solutions for semi-linear absract differential equations with not instantaneous impulses, Applied mathematics and computation, 219 (2013), 6743 - 6749.

I Podlubny, Fractional differential equations, Mathematics in Sciences and Engineering, Academic press, San Diego, 1999.

J Prüss, Evolutionary integral equations and Applications, Monographs in Mathematics, 87, Birkhäuser Verlag, Basel, 1993.

JinRang Wang, Y. Zhou, Z. Lin, On a new class of impulsive fractional differential equations, Appl. Math. and Comput., 242 (2014), $649-657$. 